\title{
Cherblanc, J., Dorais, F.-O., Tremblay, C. et Tremblay, S. (dir.). (2020). La COVID-19 : un fait social total-Perspectives historiques, politiques, sociales et humaines. Chicoutimi, Québec : Groupe de recherche et d'interventions régionales, 169 p.
}

\section{Christian Bélanger ${ }^{\mathrm{a}}$}

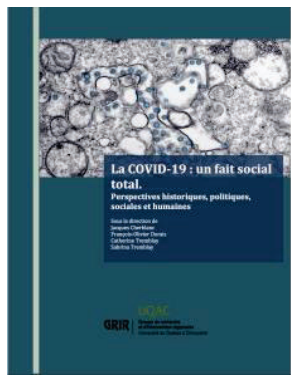

Cet ouvrage collectif constitue un apport aux réflexions interdisciplinaires prenant place au sein de l'espace public à propos des impacts de la pandémie de COVID-19. Celle-ci nous place devant de nombreux enjeux et défis aussi variés que multiples. Elle constitue un objet de préoccupation pour l'ensemble des organisations et de nos sociétés. Toute démarche cherchant à comprendre et à expliquer la crise sanitaire qu'entraine la pandémie appelle à une analyse en fonction d'une approche systémique. La COV ID-19 : un fait social total est un ouvrage souscrivant à cette approche. Par le biais de l'interdisciplinarité, il propose d'apporter des réponses aux questionnements qu'entraine la pandémie, de même que la création d'un espace de dialogue.

Fruit d'une collaboration des chercheurs du Département de sciences humaines et sociales (DSHS) de l'Université du Québec à Chicoutimi (UQAC), l'ouvrage présente les contributions de plus de 40 intervenants. La préface est de Gérard Bouchard, qui y formule quelques réflexions à propos de la pandémie touchant les thèmes de la peur, du retour de la nation, des inégalités et des différents horizons d'analyse du sujet. L'introduction de Jacques Cherblanc, François-Olivier Dorais, Catherine Tremblay et Sabrina Tremblay rappelle l'importance de la crise sanitaire. En tant que fait social total, celle-ci exerce une influence sur les facteurs à l'origine des dynamiques sociales de même que sur l'évolution probable de ces dernières. Puisque la pandémie caractérise les aspects sociaux et humains des différentes collectivités, la considération de ces aspects est essentielle à la compréhension et à l'explication des incidences de la pandémie. Cela suppose la prise en compte de différents apports du champ disciplinaire des sciences humaines et sociales. L'ouvrage collectif comprend un total de 15 textes répartis en 4 parties.

\section{Première partie : la dimension historique de la pandémie (3 textes)}

Le premier texte est d'Érik Langevin et de François Guérard. Il traite de l’influence des crises sanitaires passées dans la réaction des membres des Premières Nations à la pandémie actuelle de COVID-19. Dès l'époque de la Nouvelle-France, le contact de ces derniers avec la population en provenance de l'Europe a contribué à la transformation de leur environnement sanitaire. L'existence d'une mémoire historique de ces expériences contribue à expliquer la réaction des membres des Premières Nations face à la pandémie actuelle.

François Guérard et François-Olivier Dorais nous convient à une réflexion à propos de la crise sanitaire actuelle par le biais d'une comparaison avec celle de la grippe espagnole de 1918. Ils illustrent la situation québécoise et s'attardent à celle du Saguenay-Lac-Saint-Jean. Pour des raisons qui diffèrent, les deux crises

a Ph. D., chargé de cours, Université du Québec à Chicoutimi 
sanitaires mettent en lumière l'importance du rôle des médias à titre de vecteur informationnel de même que des impacts d'une approche populiste aux dépens d'une approche scientifique. Elles ont aussi été l'occasion de remises en question des pratiques en matière d'organisation des services de santé publique.

Le troisième texte, sur la dimension historique de la pandémie, est de Suzanne Tremblay. Il porte sur la place que la peur occupe en situation de crise sanitaire et sur les incidences qu'elle engendre au sein de nos sociétés modernes. L'auteure souligne que ce sentiment n'est pas un élément caractérisant uniquement la pandémie actuelle. Toutefois, à l'aide des propos des sociologues Ulrick Beck et Zygmunt Bauman, elle met de l'avant la possibilité de l'instrumentalisation de la peur afin de définir les enjeux auxquels les sociétés font face à l'heure de la COVID-19. Les incidences de cette situation ne sont pas sans conséquence, notamment en matière de repli sur soi, d'isolationnisme et d'intolérance.

\section{Deuxième partie : la dimension économique et politique de la pandémie (3 textes)}

Pierre-André Tremblay aborde les impacts appréhendés de la crise sanitaire sur l'évolution de différents champs composant la société. Il traite des formes de la sociabilité, de la spatialité des liens sociaux de même que de la légitimité du savoir dans l'organisation sociale. Celles-ci auraient pour dénominateur commun la surveillance étatique des rapports qu'entretiennent les individus, ce qui illustre dès lors une modification du rapport entre l'État et la société engendrée par la pandémie.

Le deuxième texte est de Romuald Jamet et de Kim Truchon. Il traite de l'émergence des modes de gouvernance fondés sur l'utilisation de l'intelligence artificielle dans le cadre de la pandémie. Pour ce faire, ceux-ci impliquent une multitude de technologies permettant la formulation d'algorithmes prédictifs des comportements. La situation québécoise contribue à mettre en évidence les débats et les enjeux vécus à l'échelle planétaire, notamment en ce qui a trait aux impacts possibles et appréhendés des applications de traçage.

Le troisième texte est de Michel Roche, dont les propos mettent de l'avant le rôle crucial de l'État-nation dans le cadre de la formulation d'une réponse aux conséquences de la pandémie de COVID-19. L'auteur prend le contre-pied du discours idéologique néolibéral ayant prévalence depuis un certain nombre de décennies. Par le biais de ses interventions, il apporte des réponses aux impacts de la crise sanitaire dans plusieurs domaines, ce qui peut amener à la formulation de questionnements légitimes en ce qui a trait à un retour (dans la durée) de la prédominance du système social sur celui de l'économie dans nos sociétés.

\section{Troisième partie : les réalités sociales et territoriales en temps de pandémie (4 textes)}

D'abord, Sabrina Tremblay aborde la question des dynamiques et des composantes territoriales auxquelles nous ramène la pandémie. L'une d'elles est le développement de la territorialité (sentiment d'appartenance) au regard de pratiques sociales réactivées et d'un changement d'échelle permettant d'apporter une réponse aux besoins de base de la population. À cela s'ajoute la confrontation entre une centralisation des décisions en matière de gestion de la santé publique et une perte sur la capacité d'agir des communautés afin de favoriser ses choix et son développement en la matière. La perte de pouvoir des individus en temps de crise sanitaire pose aussi la question du retour ou de la recherche de ce même pouvoir dans le cadre des actions de contestation des autorités publiques.

Le deuxième texte est de Marcelle Dubé, qui traite des impacts de la crise sanitaire sur les pratiques de médiation culturelle et des incidences que cela engendre pour certains organismes culturels de la région du SaguenayLac-Saint-Jean. L'avènement de la pandémie a entrainé de nombreux changements en ce qui a trait aux pratiques de travail mises de l'avant par différentes organisations. À cela se sont ajoutées la perte des liens établis avec les populations qu'elles rejoignaient ainsi que la migration des pratiques de médiation culturelle vers le numérique. Pour l'auteure, de nombreux défis restent à venir pour le milieu culturel en ce qui a trait à la mise sur pied et la proposition de différentes activités. 
À l'aide du cas du Centre de solidarité internationale du Saguenay-Lac-Saint-Jean, Marie Fall aborde les incidences de la pandémie sur l'action qu'exercent les organismes de coopération internationale basés en région. L'exécution des projets de ces organismes implique généralement un contexte de stabilité et de mobilité, lequel est remis en question par la crise sanitaire. Cela entraine une réorganisation de certains projets terrain vers une réponse d'urgence à la pandémie. Toutefois, cela n'entraine pas l'arrêt de certains projets dont la pertinence est accentuée par le contexte pandémique. L'absence de mobilité à l'international pour les stagiaires de différents programmes amène à se questionner sur les impacts en matière de solidarité internationale.

Le dernier texte de cette partie est de Marc-Urbain Proulx, qui met de l'avant une réflexion ayant trait à la relance post-pandémie des régions périphériques du Québec. Pour ce qui est de la région du Saguenay-LacSaint-Jean, l'auteur rapporte qu'il faudrait envisager de nouvelles options en matière de développement. S'inscrivant de plain-pied dans le cadre de la transition énergétique, l'énergie renouvelable est des plus prometteuses. L'auteur met de l'avant les ressources et les compétences du Saguenay-Lac-Saint-Jean, puis formule une proposition qui permettrait d'agir en ce sens, soit celle de la création d'une commission régionale de l'énergie renouvelable.

\section{Quatrième partie : les impacts sociaux de la pandémie (5 textes)}

D’abord, Danielle Maltais, Taha Abderrafie Moalla, Ève Pouliot, Christiane Bergeron-Leclerc et Jacques Cherblanc se penchent sur les impacts de la pandémie et des mesures gouvernementales qui en ont subséquemment découlé sur la santé et le fonctionnement social de la population québécoise. La crise sanitaire constitue une catastrophe dont les conséquences s'avèrent plus accentuées pour certains groupes sociaux déjà fragilisés. Les mesures de confinement et d'atténuation des situations de propagation n'ont pas été sans conséquence. Au Québec, cela a occasionné un stress au sein de l'ensemble de la population, notamment les travailleuses de la santé. La transformation des pratiques de socialisation a pu contribuer à l'amplification de problèmes psychosociaux de même qu'à la stigmatisation et à la xénophobie à l'endroit de certains groupes de la population. S'ajoute également l'accroissement de la charge mentale des personnes, notamment celle des femmes, en lien avec une augmentation des responsabilités familiales.

Le deuxième texte est de Christiane Bergeron-Leclerc, Danielle Maltais, Jacques Cherblanc, Ève Pouliot, Jacinthe Dion, Virginie Attard et Ariane Blackburn. Il traite des impacts de la pandémie sur la santé biopsychosociale et spirituelle des étudiants et des employés de l'Université du Québec à Chicoutimi (UQAC). Les données utilisées $[\mathrm{N}=442]$ proviennent d'une étude longitudinale menée au sein de l'ensemble des composantes du réseau de l'Université du Québec. L'existence des impacts de la crise sanitaire sur l'ensemble de la population de l'UQAC est certaine. Ceux-ci se sont manifestés de différentes manières. Toutefois, la question de la santé psychologique retient l'attention, particulièrement en ce qui concerne les étudiants. Les informations mettent en évidence le degré de vulnérabilité de cette partie importante de la population de l'institution.

Le texte de Catherine Flynn, Marie-Christine Brault, Ève Pouliot et leurs collaborateurs aborde la question des inégalités vécues par les étudiantes et étudiants de l’Université du Québec à Chicoutimi (UQAC) dans le cadre de la crise sanitaire, de même que des impacts des mesures de distanciation sur leur santé. Les données utilisées $[\mathrm{N}=123]$ proviennent d'une étude mixte menée auprès de six établissements du réseau de l'Université du Québec. La pandémie a contribué à l'accentuation des défis que vivaient déjà certains groupes au sein de la population étudiante. Les impacts financiers ont été plus accrus chez les femmes de même que chez les étudiantes et étudiants en situation de handicap. Ils l'ont également été chez les étudiantes et étudiants autochtones ou racisés. Les impacts sur la santé mentale tendent à être plus accentués chez certains groupes de la population étudiante, dont les femmes et les personnes en situation de handicap, notamment en fonction de l'augmentation de la charge de travail à laquelle celles-ci sont appelées à faire face, de même que des changements relatifs à leur situation. 
Guitté Hartog, Monica Carrasco Gomez et Edith Kauffer mettent de l'avant les réponses formulées par le biais d'organisations civiles mexicaines afin de faire face à l'accroissement appréhendé de la violence basée sur le genre dans le contexte de la crise sanitaire. La violence basée sur le genre est une réalité déshumanisante pouvant se manifester de différentes manières. La banalisation des cas de violence conjugale vécue par les femmes de la part du président du pays a engendré une campagne de visibilité de cette réalité menée par des groupes communautaires féministes. Dans l'État du Chiapas, une conséquence de la pandémie a été l'augmentation du fardeau des femmes et l'aggravation des facteurs de violence. Des ateliers d'accompagnement des femmes de même que des réseaux d'entraide et de protection ont été créés afin d'y apporter des réponses. Des campagnes ont été tenues à l'échelle nationale afin d'inciter les hommes à développer des façons saines de mieux vivre ensemble. À cela se sont ajoutés le développement de différentes ressources d'aide et des activités dans le cadre de programmes visant à soutenir les jeunes LGBTQ+.

Le dernier texte est de Marc Jean, qui nous convie à une réflexion ayant trait aux enjeux éthiques que peut soulever une prise de décision dans le cadre des soins administrés à la personne. Celle-ci peut être la source de nombreux dilemmes et défis au sein d'une organisation, de même que pour les personnes soignantes et celles formulant une demande en ce sens. À l'aide d'un exemple, l'auteur fait état du type de démarche qui peut être effectué afin de conjuguer les différentes attentes individuelles et collectives des personnes impliquées.

L'ouvrage La COVID-19: un fait social total - Perspectives historiques, politiques, sociales et bumaines est pertinent et susceptible d'enrichir la compréhension ainsi que les connaissances de toute personne à propos des enjeux et des défis que soulève la crise sanitaire actuelle. 